\title{
Influenza-like illness in residential care homes: a study of the incidence, aetiological agents, natural history and health resource utilisation
}

\author{
D S Hui, ${ }^{1}$ J Woo, ${ }^{1,2}$ E Hui, ${ }^{2}$ A Foo, ${ }^{1}$ M Ip, ${ }^{3}$ K-W To, ${ }^{1}$ E S C Cheuk, ${ }^{3}$ W-Y Lam, ${ }^{3}$ A Sham, ${ }^{4}$ \\ P K S Chan ${ }^{3}$
}

- Figs 1 and 2 are published online only at http://thorax.bmi. com/content/vol63/issue8

1 Department of Medicine and Therapeutics, The Chinese University of Hong Kong, Prince of Wales Hospital, Shatin, New Territories, Hong Kong;

2 Department of Medicine and Geriatrics, Shatin Hospital, New Territories, Hong Kong;

${ }^{3}$ Department of Microbiology, The Chinese University of Hong Kong, Prince of Wales Hospital, Shatin, New Territories, Hong

Kong: ${ }^{4}$ Department of

Community and Family

Medicine, The Chinese

University of Hong Kong, Prince of Wales Hospital, Shatin, New Territories, Hong Kong

Correspondence to: Professor D S Hui, Department of Medicine amd Therapeutics, The Chinese University of Hong Kong, 30-32 Ngan Shing St,

Prince of Wales Hospital, Shatin, New Territories, Hong Kong; dschui@cuhk.edu.hk

Received 21 September 2007 Accepted 9 January 2008

Published Online First

1 February 2008

\section{ABSTRACT}

Background: Influenza-like illness (ILI) among elderly people living in residential care homes (RCHEs) is a common cause for hospitalisation. A study was undertaken to examine the incidence, underlying aetiology, natural history and associated healthcare resource utilisation related to ILI in the RCHE population.

Methods: A prospective study of ILI in four RCHEs in Shatin, Hong Kong was conducted from April 2006 to March 2007. Each RCHE was monitored daily for the occurrence of ILI and followed up until resolution of illness or death. Clinical features were recorded and sputum, nasopharyngeal aspirate, blood and urine specimens were examined for underlying aetiology.

Results: 259 episodes of ILI occurred in 194 subjects, with mild peaks in winter and summer, over a sustained level throughout the year. The infectious agent was identified in $61.4 \%$ of all episodes, comprising bacterial infection in $53.3 \%$ and viral in $46.7 \%$. Multiple infections occurred in $16.2 \%$ of subjects. The most frequent organism was Streptococcus pneumoniae, followed by respiratory syncytial virus, Pseudomonas aeruginosa, metapneumovirus and parainfluenza virus types 1 and 3 . Clinical features did not vary according to the underlying aetiology, the common presenting features being a decrease in general condition, cognitive and functional deterioration, and withholding of food in addition to fever and respiratory symptoms. Overall, mortality at 1 month/ discharge was $9.7 \%$. Infection with methicillin-resistant Staphylococcus aureus, low body mass index and poor function predisposed to mortality. No association was observed between influenza vaccination status and underlying aetiology, clinical features or outcome.

Conclusions: The clinical presentation of ILI is nonspecific and is mainly due to bacterial and viral infections other than influenza in the RCHE population.

Influenza-like illness (ILI) among elderly people living in residential care homes (RCHEs) is a public health concern as it may potentially give rise to outbreaks resulting in admission to hospital. It is difficult to identify clinically whether ILI is due to outbreaks of emerging viral infections with serious consequences such as $\mathrm{H} 5 \mathrm{~N} 1$, or other more "benign" infections such as seasonal influenza or respiratory syncytial virus (RSV). Currently, ILI in RCHEs is monitored by the Centre for Health Protection (CHP) in Hong Kong by passive surveillance, relying on a staff member of each RCHE acting as an infection control officer. Other preventive strategies include free annual seasonal influenza vaccination and oseltamivir chemoprophylaxis for residents in RCHEs in which an influenza outbreak has occurred.

Influenza vaccination has been shown to be effective in reducing hospital admissions during an influenza epidemic, particularly in institutional settings, and may reduce half of the deaths due to influenza. ${ }^{2}$ However, this strategy will only be effective if the ILI episode is caused by influenza. Furthermore, a recent study has queried the efficacy of influenza vaccination for residents of old peoples' homes in Hong Kong. ${ }^{3}$ There is a suggestion that, in old peoples' homes, respiratory infections are caused mostly by pathogens other than influenza virus, ${ }^{4}$ whereas RSV could represent a disease burden similar to that of seasonal influenza $A$ in a population in which the prevalence of vaccination against influenza is high (eg, in elderly or high-risk adults. ${ }^{5}$ Oseltamivir chemoprophylaxis is also recommended but it is offered only to those aged $<80$ years without chronic diseases and normal renal function documented in recent months. ${ }^{6}$ Assuming that influenza is the cause of most cases of ILI, this local policy appears to be empirical and not evidence-based but, at the same time, consumes significant healthcare resources.

In reality, ILI is merely a description of a clinical presentation consisting of fever $\geqslant 37.8^{\circ} \mathrm{C}$ or an acute deterioration in physical or mental ability, plus either new onset of one or more respiratory symptoms or an acute worsening of a chronic condition involving respiratory symptoms. ${ }^{7}$ It may be caused by bacterial or any viral infections of which influenza is only one of many. Furthermore, the underlying cause may not be infective but a consequence of aspiration, a common occurrence in the frail elderly population. ${ }^{8}$ Figures based on passive surveillance may underestimate the true incidence. ${ }^{\text {Th }}$ This study aimed to document the incidence of ILI, the underlying infective aetiology, the natural history and associated healthcare resource utilisation over a 1-year period.

\section{METHODS}

\section{Residential care homes for the elderly (RCHEs)}

RCHEs supported by the Hong Kong Hospital Authority's Community Geriatric Outreach Teams (CGAT) in the Shatin district were studied. Two were subsidised (Sage and Caritas) with 204 and 256 residents, respectively, and two were private (Shui On and Cambridge) with 221 and 91 residents, respectively. Infection control policies recommended by the CHP were implemented in all RCHEs and were complied with by reports. 
Research staff liaised with the nurse of the CGAT or the Visiting Medical Officer (VMO) responsible for the homes to identify residents with ILI on a daily basis. An ILI episode was defined as fever $\geqslant 37.8^{\circ} \mathrm{C}$ measured using a digital aural thermometer or an acute deterioration in physical or mental ability, plus either new onset of one or more respiratory symptoms or an acute worsening of a chronic condition involving respiratory symptoms. ${ }^{7}$ Specimens were collected within the first 2 days of illness and paired serology 2 weeks later, whether the patients were still in hospital or had returned to the RCHE. A follow-up visit was carried out after recovery of illness.

\section{Investigations}

All subjects with clinical features fulfilling the inclusion criteria for ILI underwent the following investigations:

- Sputum sample

- Rapid multiplex nested PCR

- Paired serum samples

- Urine sample

\section{Sputum sample}

Sputum samples were used for:

- Routine bacterial culture which covers Streptoccocus pneumoniae, Pseudomonas sp, Haemophilus influenzae, Moraxella catarrhalis and other likely pathogens.

- Mycobacterial culture for tuberculosis (restricted to those with prolonged respiratory symptoms such as cough for $\geqslant 2$ weeks, systemic symptoms of weight loss or hospital admission).

\section{Rapid multiplex nested PCR}

A rapid multiplex nested PCR was undertaken to detect respiratory pathogens. Five groups of multiplex nested-PCR assays targeting 21 respiratory viruses and bacteria were developed. Each detected four viruses and/or bacteria: group 1 comprised influenza A and B group-specific and subtypes H1N1, H3N2, H5N1-specific primers; group 2 comprised parainfluenza 1, 2, 3, $4 \mathrm{a}$ and $4 \mathrm{~b}$; group 3 comprised RSV A and B, human rhinovirus and enterovirus; group 4 comprised human coronavirus (CoV)OC43, -229E, -SARS-CoV and human metapneumovirus (hMPV); and group 5 comprised Mycoplasma pneumoniae, Chlamydophila pneumoniae, Legionella pneumophila and adenovirus. ${ }^{10}$

Nasopharyngeal aspirates were collected and subjected to RNA and DNA extraction. Both viral RNA and DNA were extracted by spin columns (OIAamp MinElute Virus Spin Kit, Qiagen, USA) according to the manufacturer's recommendations. RNA extracted was converted to cDNA by reverse transcriptase (Superscript III Reverse Transcriptase, Invitrogen, Carlsbad, California, USA). All the DNA and cDNA were used immediately for the five groups of nested PCR as described previously.

\section{Paired serum samples}

A paired serum sample was used for atypical pneumonia serological examination including influenza A, influenza B, parainfluenza 1, 2, 3, RSV, adenovirus, mycoplasma, $L$ pneumophila, Coxiella burnetii, Chlamydia spp. The methodology used was the complement fixation test.

\section{Urine sample}

A urine sample (for hospitalised cases) was taken to detect $L$ pneumophila serogroup 1 and $S$ pneumoniae antigens (BinaxNOW, Maine, USA).
A chest radiograph was performed in subjects with a cough lasting $\geqslant 2$ weeks and as part of the clinical management as indicated for other cases.

In determining the aetiology of ILI, the following criteria were used: positive culture from sputum, blood or nasopharyngeal aspirate; positive urinary antigen for $S$ pneumoniae or $L$ pneumophila serogroup 1; positive PCR identification; serology of fourfold rise or a single titre of 80 or above, and a positive IgM.

Subjects with ILI were managed by the VMO or a doctor from the CGAT in the RCHE. Treatment included antipyretics, antibiotics, antiviral drugs depending on the clinical presentation, attention to hydration and nutrition. Any need for admission to hospital was the decision of the attending doctor and also RCHE staff who might have difficulty in providing additional care or monitoring of the patient.

\section{Documentation of natural history of illness}

The following data were documented for each patient: age, sex, past medical history, past vaccination history, co-existing diseases, pre-morbid functional status, presenting symptoms and signs of the illness, investigation results, treatment, allied health services, duration of illness, duration of hospital stay if relevant, mortality as a result of the ILI episode, any functional or cognitive consequences as measured by the Barthel index (BI) and Mini Mental State Examination (MMSE). The activities of daily living (ADL) score was assessed using the BI which covers various dimensions of activities of daily living, with a total score of $20{ }^{11}$ In general a score of 20 is considered to represent independence, 15-19 represents mild to moderate functional limitation and a score of $<15$ represents severe limitation. ${ }^{12}$ The ADL score before illness was obtained from the records of the RCHE and the staff.

\section{Sample size calculation}

Currently, about $10 \%$ of residents of RCHEs have an ILI episode over a 1-year period. Assuming a SD of 4\%, a total of 217 subjects or ILI episodes would need to be studied. ILI episodes in Hong Kong generally occur in two major peaks in late summer and winter, in addition to low level background occurrences. Assuming a $10 \%$ incidence, the number of episodes covering the two peaks over a 1 -year period would be $2 \times 0.1 \times 1000=200$. Additional episodes of 80 during the other periods would be expected. Assuming 10\% were missed, the remaining episodes that could be studied would be $0.9 \times 280=252$.

\section{Statistical analysis}

The variations in the number of ILI episodes according to season and the prevalence of different aetiologies of ILI in RCHE are shown. The number of episodes each month was divided by the total number of residents in each RCHE to calculate the incidence, and the differences between the four seasons (December-February, March-May, June-August, SeptemberNovember) and between the four homes were examined by the $\chi^{2}$ test. The clinical presentation, natural history and outcome according to the different causes of ILI were examined and tested for statistically significant differences using a $\chi^{2}$ test for categorical variables and ANOVA for continuous variables. Factors associated with mortality were examined using univariate analysis and the results presented as unadjusted odds ratios (ORs) per unit OR for continuous variables (body mass index, MMSE and ADL score) and per year for age, assigning an OR of 1 to the factor level used for comparison. The average cost of an ILI episode to the hospital authority was estimated in terms of bed-days occupied and drug costs using figures supplied by the 
Table 1 Incidence of influenza-like illness (ILI) in four residential care homes in Shatin over a 1-year period

\begin{tabular}{lccccc}
\hline & $\begin{array}{l}\text { Caritas } \\
(\mathbf{n}=\mathbf{2 5 6})\end{array}$ & $\begin{array}{l}\text { Sage } \\
(\mathbf{n}=\mathbf{2 0 4 )}\end{array}$ & $\begin{array}{l}\text { Shui } \mathbf{0 n} \\
(\mathbf{n}=\mathbf{2 2 1})\end{array}$ & $\begin{array}{l}\text { Cambridge } \\
(\mathbf{n}=\mathbf{9 0})\end{array}$ & $\begin{array}{l}\text { Total } \\
(\mathbf{n}=\mathbf{7 7 1})\end{array}$ \\
\hline $\begin{array}{l}\text { Total number with ILI } \\
\text { Date of onset }\end{array}$ & $83(32.4)$ & $62(30.4)$ & $86(38.9)$ & $28(31.1)$ & $259(33.6)$ \\
$\quad$ & & & & \\
April & $1(0.4)$ & $8(3.9)$ & $11(5.0)$ & $6(6.7)$ & $26(3.4)$ \\
May & $5(2.0)$ & $5(2.5)$ & $11(5.0)$ & $1(1.1)$ & $22(2.9)$ \\
June & $7(2.7)$ & $10(4.9)$ & $4(1.8)$ & $5(5.6)$ & $26(3.4)$ \\
July & $10(3.9)$ & $7(3.4)$ & $7(3.2)$ & 0 & $24(3.1)$ \\
August & $7(2.7)$ & $1(0.5)$ & $7(3.2)$ & $2(2.2)$ & $17(2.2)$ \\
September & $8(3.1)$ & $5(2.5)$ & $4(1.8)$ & $1(1.1)$ & $18(2.3)$ \\
October & $11(4.3)$ & $3(1.5)$ & $7(3.2)$ & $2(2.2)$ & $23(3.0)$ \\
November & $9(3.5)$ & $4(2.0)$ & $3(1.4)$ & $1(1.1)$ & $17(2.2)$ \\
December & $3(1.2)$ & $2(1.0)$ & $4(1.8)$ & $2(2.2)$ & $11(1.4)$ \\
Jan & $2(0.8)$ & $4(2.0)$ & $7(3.2)$ & $2(2.2)$ & $15(1.9)$ \\
Feb & $5(2.0)$ & $5(2.5)$ & $3(1.4)$ & $2(2.2)$ & $15(1.9)$ \\
Mar & $15(5.8)$ & $8(4.0)$ & $18(8.2)$ & $4(4.4)$ & $45(5.9)$ \\
April-June & $13(5.1)$ & $23(11.3)$ & $26(11.8)$ & $12(13.3)^{*}$ & $74(9.6)$ \\
July-Sept & $25(9.8)$ & $13(6.4)$ & $18(8.1)$ & $3(3.3)$ & $59(7.7)$ \\
Oct-Dec & $23(9.0)$ & $9(4.4)$ & $14(6.3)$ & $5(5.6)$ & $51(6.6)$ \\
Jan-March & $22(8.6)$ & $17(8.4)$ & $28(12.7)$ & $8(8.9)$ & $75(9.7)$ \\
\hline Data are shown as n (\%). & &
\end{tabular}

hospital authority applicable to Shatin. The presence and titres of anti-influenza antibodies were correlated with influenza vaccination status using a $\chi^{2}$ test and an ordinal trend measure.

\section{RESULTS}

\section{Incidence of ILI by RCHEs over a 1-year period}

A total of 259 episodes of ILI occurred in 194 subjects; $77.8 \%$ of these subjects had one episode, $4.4 \%$ had two, $4.6 \%$ had three, $2.6 \%$ had four and one subject had five episodes. The number of ILI episodes and the incidence of ILI for each RCHE (calculated as the number of ILI episodes divided by the total number of residents) are shown in table 1. Overall, the incidence was slightly higher during January-March and April-June, although

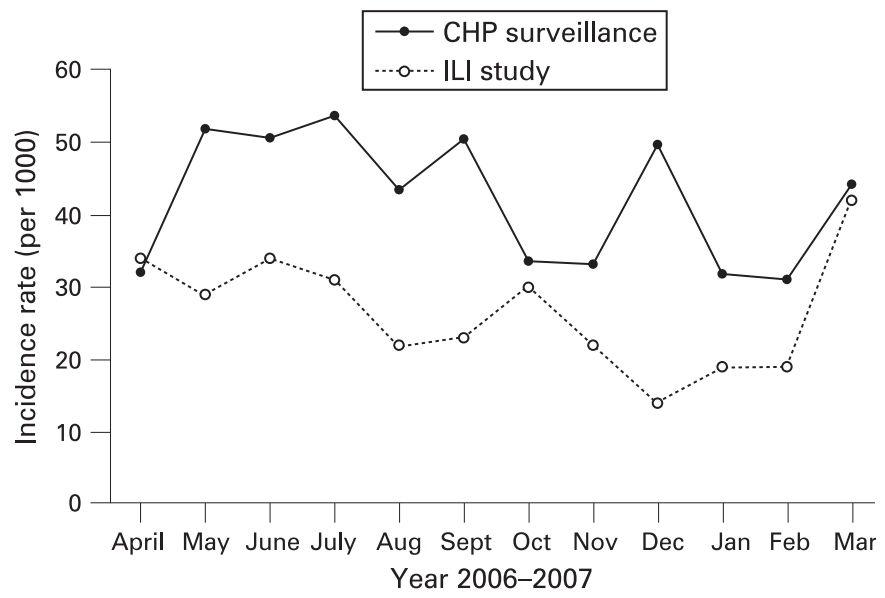

Figure 1 Comparisons of fever surveillance at sentinel residential care homes for the elderly in 2006-7. There was close correlation only for March and April, which are recognised as peak months for viral infections such as influenza. The results suggest that, during certain months, a common infectious agent affecting the community at large may be the more important underlying aetiology for an influenza-like illness (ILI), while there might be greater variability in the underlying aetiology depending on the characteristics of the resident and residential care home at other times. CHP, Centre for Health Protection. overall the incidence in different seasons did not reach statistical significance. However, the variation in incidence differed among the RCHEs, with all documenting the highest incidence in April-June with the exception of Caritas.

To examine whether the pattern of ILI was similar to the figures provided by the CHP for sentinel RCHEs, the incidence figure was converted to number/1000 (see fig. 1 in online data supplement). In comparison with the CHP data for the community (see fig 2 in online data supplement), the month-to-month variation showed some similarity with the rates from private doctors and general outpatient clinics in the rise in June and February-March, although the magnitude was less. The incidence for RCHEs was also lower than for the general community. These findings are compatible with the spread of infectious agents from the community to RCHEs via staff and visitors during episodes when infections are prevalent in the community, accounting for some of the variations in ILI in RCHEs.

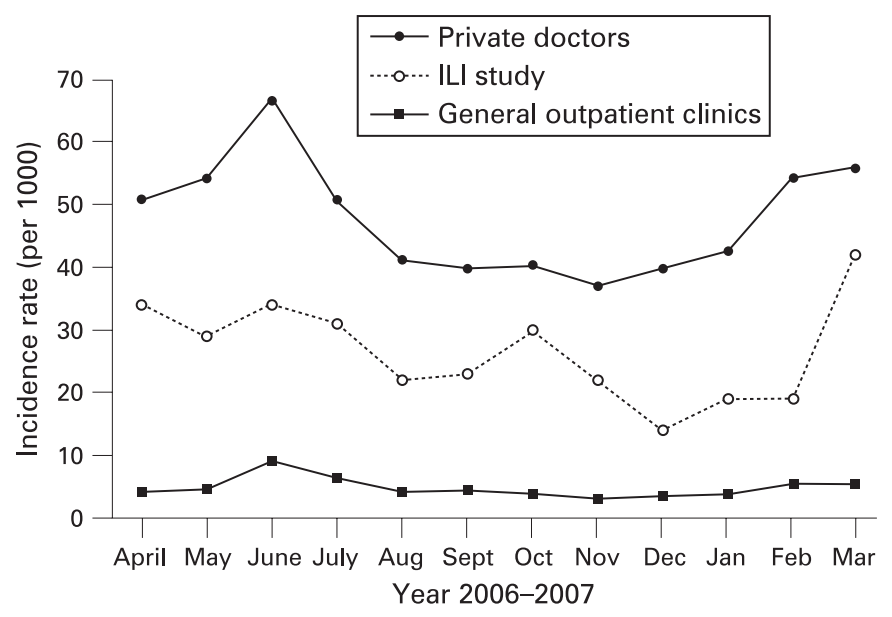

Figure 2 Comparisons of influenza-like illness (ILI) surveillance at sentinel private doctors and general outpatient clinics, 2006-7. 


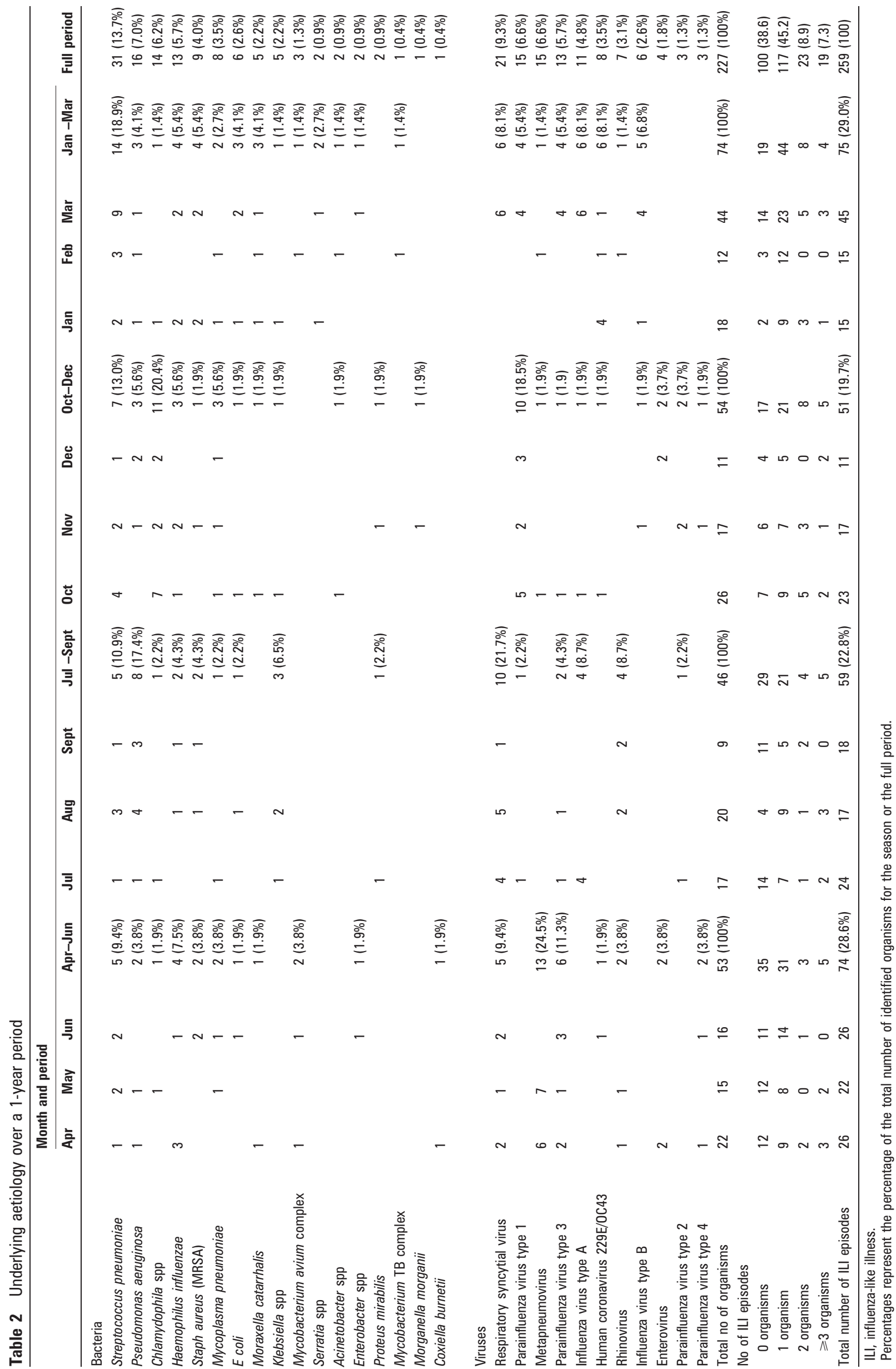


Table 3 Clinical characteristics of patients suffering an influenza-like illness (ILI) episode*

\begin{tabular}{|c|c|}
\hline & Total $(n=259$ \\
\hline \multicolumn{2}{|l|}{ Age (years) } \\
\hline$<80$ & $61(23.6)$ \\
\hline $80-89$ & $118(45.6)$ \\
\hline $90+$ & $80(30.9)$ \\
\hline Mean (SD) & $84.9(8.9)$ \\
\hline \multicolumn{2}{|l|}{ Sex } \\
\hline Male & $115(44.4)$ \\
\hline Female & $144(55.6)$ \\
\hline BMI $\left(\mathrm{kg} / \mathrm{m}^{2}\right)$ & $22.2(5.0)$ \\
\hline \multicolumn{2}{|l|}{ Presenting symptoms } \\
\hline Fever & $230(88.8)$ \\
\hline Productive cough & $171(66.0)$ \\
\hline Non-productive cough & $71(27.5)$ \\
\hline Cough with white phlegm & $104(40.5)$ \\
\hline Cough with yellow phlegm & $96(37.4)$ \\
\hline Cough with blood & $5(1.9)$ \\
\hline Shortness of breath & $159(61.6)$ \\
\hline Wheeze & $59(23.0)$ \\
\hline Decrease in general condition & $182(70.5)$ \\
\hline \multicolumn{2}{|l|}{ Flu vaccination } \\
\hline Within past 6 months & $124(47.9)$ \\
\hline Within past 12 months but not 6 months & $108(41.7)$ \\
\hline Given oseltamivir chemoprophylaxis & $2(0.8)$ \\
\hline Presence of delirium & $11(4.2)$ \\
\hline \multicolumn{2}{|l|}{ MMSE } \\
\hline At beginning of illness & $16.4(6.3)$ \\
\hline At end of illness & $17.7(6.9)$ \\
\hline Deterioration in $\mathrm{ADL}$ score & $169(65.5)$ \\
\hline \multicolumn{2}{|l|}{ ADL score } \\
\hline Before illness (from cases) & $6.5(5.9)$ \\
\hline During illness & $3.9(4.5)$ \\
\hline At end of illness & $6.0(5.8)$ \\
\hline Hospital admission & $226(87.3)$ \\
\hline Mean (SD) duration of stay (days) & $14.6(12.9)$ \\
\hline Outcome died & $25(9.7)$ \\
\hline $\begin{array}{l}\text { Mean (SD) duration of illness if not admitted (days) } \\
(\mathrm{n}=33)\end{array}$ & $8.8(5.2)$ \\
\hline Mean (SD) duration of fever (days) & $2.4(3.4)$ \\
\hline Oxygen used & $162(63.0)$ \\
\hline Intravenous antibiotics & $229(87.0)$ \\
\hline Intravenous fluids & $167(65.0)$ \\
\hline Withholding food & $146(56.8)$ \\
\hline Mean (SD) duration of withholding food (days] & $4.1(6.4)$ \\
\hline \multicolumn{2}{|l|}{ Use of allied health service } \\
\hline Speech therapist & $100(39.1)$ \\
\hline Occupational therapist & $3(1.2)$ \\
\hline Physiotherapist & $183(71.5)$ \\
\hline Dietitian & $55(21.5)$ \\
\hline \multicolumn{2}{|l|}{ Abnormal chest signs } \\
\hline Wheeze & $44(17.2)$ \\
\hline Dullness & $12(4.7)$ \\
\hline Reduced air entry & $51(20.0)$ \\
\hline Bronchial breathing & $9(3.5)$ \\
\hline Crepitations & $139(54.5)$ \\
\hline \multicolumn{2}{|l|}{ Chest radiography } \\
\hline III-defined shadow & $82(36.8)$ \\
\hline Consolidation & $46(20.7)$ \\
\hline Pleural effusion & $13(5.9)$ \\
\hline New lesions compared with previous radiograph & $16(7.3)$ \\
\hline
\end{tabular}

$\mathrm{ADL}$, activities of daily living; $\mathrm{BMI}$, body mass index; MMSE, Mini Mental State Examination.

*Data given for ILI episodes, and more than one episode may occur in the same subject. $\uparrow$ The figure may not add up to $100 \%$ as patients may have more than one symptom.

\section{Underlying aetiology of ILI in RCHEs}

In this study, nasopharyngeal aspirates were processed in 256 of 259 episodes of ILI. Sputum for bacterial cultures was obtained in only 134 episodes. An infectious agent was identified in $61.4 \%$ of ILI episodes (table 2). Bacterial infections accounted for $53.3 \%$ and viruses were identified in $46.7 \%$. In 42 ILI episodes (16.2\%) two or more organisms were identified, and 27 episodes $(10.4 \%)$ had positive viral-bacterial combinations. The top five causes in order of frequency were $S$ pneumoniae, RSV, $P$ aeruginosa, parainfluenza virus type 1 and hMPV. Seasonal variations in the organisms prevalent for ILI was observed: hMPV and parainfluenza virus type 3 from April to June, RSV and $P$ aeruginosa from July to September, Chlamydophila sp, parainfluenza virus type 1 and $S$ pneumoniae from October to December and $S$ pneumoniae, RSV, influenza virus type A and human coronaviruses from January to March. For the latter period, RSV and coronavirus infections were equally prevalent as influenza A. In general, viral infections showed more seasonal variations than bacterial infections.

Among the bacterial infections, $S$ pneumoniae was the commonest during the autumn and winter months. P aeruginosa was significant in the summer months, whereas Staphylococcus aureus (including methicillin-resistant $S$ aureus (MRSA)) was among the top five bacteria. With the use of rapid molecular methods for detection, ${ }^{10}$ atypical organisms with $C$ pneumoniae and $M$ pneumoniae also contributed frequently among bacterial aetiologies. There were no marked differences in the frequency of organisms between the four institutions.

\section{Clinical features and natural history}

The results for all RCHEs are combined as there were no significant differences between them (table 3). The mean age of the patients was 85 years, with slightly more women than men. Presentation with "decrease in general condition" in terms of cognition, $\mathrm{ADL}$ and/or mobility was observed in $71 \%$. Decline in physical function occurred in about $66 \%$ of patients as a result of the illness, while cognitive decline was also observed. $87 \%$ of the ILI episodes resulted in hospital admission despite the availability of healthcare consultation, investigations and drug treatment on site.

No particular clinical features were identified for any particular organism. Mortality was $9.7 \%$. Factors associated with mortality were withholding of food (OR 3.37; 95\% CI 1.22 to 9.27); lower BMI, lower ADL score during illness and the presence of MRSA and Klebsiella spp as underlying aetiological agents (table 4). The presence or absence of the pre-morbid diseases was not associated with mortality, but we did not collect data on disease severity.

Management included intravenous antibiotic in $87 \%$ of cases, oxygen and intravenous fluids in over $60 \%$ of patients, and extensive involvement of allied health staff. Food was withheld in $57 \%$ of patients as a result of concern over possible aspiration or aspiration that might have precipitated the ILI episode.

\section{Costs to the hospital}

The costs per ILI episode are shown in table 5. In calculating overall hospital costs, the figures supplied by the hospital authority for general medical beds for the Prince of Wales (acute) and Shatin (convalescent) hospitals were used, since these were the hospitals admitting cases from the four RCHEs.

\section{Influenza vaccination status}

Approximately $90 \%$ had received influenza vaccination, half within 6 months and half at 6-12 months. The lowest 
Table 4 Factors associated with mortality

\begin{tabular}{|c|c|c|c|c|c|}
\hline & $\begin{array}{l}\text { Alive } \\
(n=234)\end{array}$ & $\begin{array}{l}\text { Died } \\
(n=25)\end{array}$ & $\begin{array}{l}\text { Total } \\
(\mathrm{n}=259)\end{array}$ & p Value: & OR $(95 \% \mathrm{Cl})$ \\
\hline \multicolumn{6}{|l|}{ Residential care home } \\
\hline Caritas & $75(32.1)$ & $8(32.0)$ & $83(32.0)$ & 0.102 & 1.00 \\
\hline Sage & $52(22.2)$ & $10(40.0)$ & $62(23.9)$ & & 1.80 (0.67 to 4.88$)$ \\
\hline Shui On & $79(33.8)$ & $7(28.0)$ & $86(33.2)$ & & 0.83 (0.29 to 2.40$)$ \\
\hline Cambridge & $28(12.0)$ & 0 & $28(10.8)$ & & \\
\hline \multicolumn{6}{|l|}{ Sex } \\
\hline Male & $104(44.4)$ & $11(44.0)$ & $115(44.4)$ & 0.966 & 1.00 \\
\hline Female & $130(55.6)$ & $14(56.0)$ & $144(55.6)$ & & 1.02 (0.44 to 2.34$)$ \\
\hline Mean (SD) age (years) & $85.0(8.9)$ & $84.2(9.3)$ & $84.9(8.9)$ & 0.674 & 0.99 (0.95 to 1.04$)$ \\
\hline \multicolumn{6}{|l|}{ Age groups } \\
\hline$<80$ & $56(23.9)$ & $5(20.0)$ & $61(23.6)$ & 0.820 & 1.00 \\
\hline $80-89$ & $107(45.7)$ & $11(44.0)$ & $118(45.6)$ & & 1.15 (0.38 to 3.48$)$ \\
\hline $90+$ & $71(30.3)$ & $9(36.0)$ & $80(30.9)$ & & $1.42(0.45$ to 4.48$)$ \\
\hline \multicolumn{6}{|l|}{ Withholding food } \\
\hline No & $106(45.7)$ & $5(20.0)$ & $111(43.2)$ & 0.014 & 1.00 \\
\hline Yes & $126(54.3)$ & $20(80.0)$ & $146(56.8)$ & & 3.37 (1.22 to 9.27 ) \\
\hline $\mathrm{BMI}\left(\mathrm{kg} / \mathrm{m}^{2}\right)$ & $22.5(5.0)$ & $18.5(3.7)$ & $22.2(5.0)$ & $<0.001$ & 0.78 (0.68 to 0.90$)$ \\
\hline \multicolumn{6}{|l|}{ MMSE } \\
\hline At beginning of illness & $16.6(3.6)$ & $14.1(4.1)$ & $16.4(6.3)$ & 0.323 & 0.94 (0.82 to 1.07 ) \\
\hline At end of illness & $17.7(6.9)$ & & & & \\
\hline \multicolumn{6}{|l|}{ Changes in ADL score } \\
\hline No & $83(35.6)$ & $6(24.0)$ & $89(34.5)$ & 0.245 & 1.00 \\
\hline Yes & $50(64.4)$ & $19(76.0)$ & $169(65.5)$ & & 1.75 (0.67 to 4.56$)$ \\
\hline \multicolumn{6}{|l|}{ ADL score } \\
\hline Before illness (from cases) & $6.6(6.0)$ & $5.0(4.7)$ & $6.5(5.9)$ & 0.127 & 0.95 (0.88 to 1.03 ) \\
\hline During illness & $4.1(4.7)$ & $1.8(1.8)$ & $3.9(4.5)$ & $<0.001$ & 0.82 (0.69 to 0.97 ) \\
\hline At end of illness & $6.0(5.8)$ & & & & \\
\hline \multicolumn{6}{|l|}{ Identified organisms } \\
\hline Streptococcus pneumoniae & $26(11.1)$ & $5(20.0)$ & $31(12.0)$ & 0.193 & 2.00 (0.69 to 5.78 ) \\
\hline Respiratory syncytial virus & $18(7.7)$ & $3(12.0)$ & $21(8.1)$ & 0.453 & $1.64(0.45$ to 6.00$)$ \\
\hline Pseudomonas aeruginosa & $15(6.4)$ & $1(4.0)$ & $16(6.2)$ & 0.634 & 0.61 (0.08 to 4.81 ) \\
\hline Parainfluenza virus type 1 & $15(6.4)$ & 0 & $15(5.8)$ & 0.192 & - \\
\hline Metapneumovirus & $14(6.0)$ & $1(4.0)$ & $15(5.8)$ & 0.687 & 0.66 (0.08 to 5.20$)$ \\
\hline Chlamydia & $13(5.6)$ & $1(4.0)$ & $14(5.4)$ & 0.744 & $0.71(0.09$ to 5.65$)$ \\
\hline Haemophilus influenzae & $11(4.7)$ & $2(8.0)$ & $13(5.0)$ & 0.473 & $1.76(0.37$ to 8.45$)$ \\
\hline Parainfluenza virus type 3 & $13(5.6)$ & 0 & $13(5.0)$ & 0.227 & - \\
\hline Influenza virus $A$ & $9(3.8)$ & $2(8.0)$ & $11(4.2)$ & 0.328 & $2.17(0.44$ to 10.67$)$ \\
\hline Staphylococcus aureus & $5(2.1)$ & $4(16.0)$ & $9(3.5)$ & $<0.001$ & $8.72(2.18$ to 34.98$)$ \\
\hline Coronavirus 229E/OC43 & $7(3.0)$ & $1(4.0)$ & $8(3.1)$ & 0.782 & 1.35 (0.16 to 11.45 \\
\hline Mycoplasma pneumoniae & $7(3.0)$ & $1(4.0)$ & $8(3.1)$ & 0.782 & $1.35(0.16$ to 11.45$)$ \\
\hline Rhinovirus & $7(3.0)$ & 0 & $7(2.7)$ & 0.381 & - \\
\hline E coli & $5(2.1)$ & $1(4.0)$ & $6(2.3)$ & 0.556 & $1.91(0.21$ to 17.01$)$ \\
\hline Influenza virus B & $6(2.6)$ & 0 & $6(2.3)$ & 0.418 & - \\
\hline Klebsiella spp & $3(1.3)$ & $2(8.0)$ & $5(1.9)$ & 0.020 & $6.70(1.06$ to 42.15$)$ \\
\hline Moraxella catarrhalis & $5(2.1)$ & 0 & $5(1.9)$ & 0.460 & - \\
\hline Enterovirus & $4(1.7)$ & 0 & $4(1.5)$ & 0.510 & - \\
\hline Others* & $18(7.7)$ & $2(8.0)$ & $20(7.7)$ & 0.828 & $1.19(0.26$ to 5.48$)$ \\
\hline \multicolumn{6}{|l|}{ Premorbid conditions } \\
\hline Hypertension & $147(62.8)$ & $14(56.0)$ & $161(62.2)$ & 0.504 & $0.8(0.3$ to 1.7$)$ \\
\hline Cerebral vascular accident & $99(42.3)$ & $10(40.0)$ & $109(42.1)$ & 0.824 & $0.9(0.4$ to 2.1$)$ \\
\hline Diabetes mellitus & $56(23.9)$ & $4(16.0)$ & $60(23.2)$ & 0.372 & $0.6(0.2$ to 1.8$)$ \\
\hline Ischaemic heart disease & $25(10.7)$ & $2(8.0)$ & $27(10.4)$ & 0.676 & $0.7(0.2$ to 3.3$)$ \\
\hline Congestive heart failure & $32(13.7)$ & $4(16.0)$ & $36(13.9)$ & 0.749 & $1.2(0.4$ to 3.7$)$ \\
\hline Dementia & $84(35.9)$ & $12(48.0)$ & $96(37.1)$ & 0.234 & $1.6(0.7$ to 3.8$)$ \\
\hline COPD & $43(18.4)$ & $6(24.0)$ & $49(18.9)$ & 0.495 & $1.4(0.5$ to 3.7$)$ \\
\hline Cancer & $12(5.1)$ & $2(8.0)$ & $14(5.4)$ & 0.546 & $1.6(0.3$ to 7.6$)$ \\
\hline $\mathrm{R} / \mathrm{T}$ feeding & $40(17.1)$ & $3(12.0)$ & $43(16.6)$ & 0.515 & $0.7(0.2$ to 2.3$)$ \\
\hline
\end{tabular}


Table 4 Continued

\begin{tabular}{llllll}
\hline & $\begin{array}{l}\text { Alive } \\
(\mathbf{n}=\mathbf{2 3 4})\end{array}$ & $\begin{array}{l}\text { Died } \\
(\mathbf{n}=\mathbf{2 5})\end{array}$ & $\begin{array}{l}\text { Total } \\
(\mathbf{n}=\mathbf{2 5 9})\end{array}$ & $\mathbf{p}$ Value $\neq$ & OR (95\% Cl) \\
\hline No of premorbid conditions & & & & & \\
0 & $13(5.6)$ & $1(4.0)$ & $14(5.4)$ & 0.471 & 1.0 \\
1 & $47(20.1)$ & $4(16.0)$ & $51(19.7)$ & & $1.1(0.1$ to 10.8) \\
2 & $74(31.6)$ & $9(36.0)$ & $83(32.0)$ & & $1.6(0.2$ to 13.6$)$ \\
3 & $65(27.8)$ & $10(40.0)$ & $75(29.0)$ & & $2.0(0.2$ to 17.0$)$ \\
$4+$ & $35(15.0)$ & $1(4.0)$ & $36(13.9)$ & & $0.4(0.02$ to 6.4$)$ \\
\hline
\end{tabular}

Data are given as $\mathrm{n}(\%)$.

$\mathrm{ADL}$, activities of daily living; BMI, body mass index; COPD, chronic obstructive pulmonary disease; MMSE, Mini Mental State Examination; R/T, Ryle's tube.

*Others included: parainfluenza virus type 2 ( 3 cases, 1 died), parainfluenza virus type 4 ( 3 cases), Mycobacterium avium complex ( 3 cases), Acinetobacter spp (2 cases), Enterobacter spp (2 cases), Proteus mirabilis (2 cases), Serratia spp (2 cases, 1 died), Coxiella burnetii (1 case), Mycobacterium tuberculosis (1 case) and Morganella morganii (1 case).

$\dagger$ The figure may not add up to $100 \%$ as patients may have more than one condition.

$\$$ The $p$ value is for the $\chi^{2}$ test of a null hypothesis proposing a value of 1 for multiple odds ratio.

percentage of unvaccinated subjects was $4 \%$ in one subsidised home, but in the other three the percentages ranged from $26 \%$ to $37 \%$. Among those vaccinated, $48 \%$ had received the vaccine within 6 months and $42 \%$ at $6-12$ months. There was no significant difference in the occurrence of influenza A infection among those who did not receive vaccination, those receiving the vaccine within 6 months or those vaccinated at 6-12 months. There were no significant differences in age, sex, clinical features, duration of hospital stay or mortality between the three vaccination status groups.

Table 6 shows the influenza A antibody titre of the day 14 serum specimen in the three vaccine status groups. The results were significantly different between the three groups, with a trend for the group receiving vaccination to have higher titres and the unvaccinated group the lowest. The more recently vaccinated group was $3.0-27.5 \%$ more likely than the less recently vaccinated group to have a higher titre. However, the size of the Somers D value indicates a high degree of overlap in titre between the three vaccine status categories. Antibody was also detected in those who did not receive vaccination.

\section{DISCUSSION}

This study has shown that ILI represents a considerable burden of disease in RCHEs, with $87 \%$ of episodes resulting in hospital admissions. The clinical presentation of ILI in the elderly people

Table 5 Mean hospital cost of influenza-like illness in HK dollars

\begin{tabular}{ll}
\hline & Total $(\mathbf{n}=\mathbf{2 5 9})$ \\
\hline Type of hospital admitted & \\
Acute only & $129(57.1 \%)$ \\
Convalescent only & $1(0.4 \%)$ \\
Both & $96(42.5 \%)$ \\
Total & 226 \\
Mean (SD) duration of stay (days) & \\
Acute hospital & $7.9(4.9)(\mathrm{n}=225)$ \\
Convalescent hospital & $15.8(12.1)(\mathrm{n}=97)$ \\
Total & $14.6(12.9)(\mathrm{n}=226)$ \\
Mean (SD) duration of oxygen use (days) & $7.2(11.9)(\mathrm{n}=162)$ \\
Mean (SD) cost for acute hospital (HK\$/day) & $20410(12614)(\mathrm{n}=225)$ \\
Mean (SD) cost for convalescent hospital (HK\$/day) & $23612(18082)(\mathrm{n}=97)$ \\
Mean (SD) cost for total hospital days (HK\$) & $30454(22662)(\mathrm{n}=226)$ \\
Mean (SD) cost of oxygen used (HK\$/day) & $150(236)(\mathrm{n}=162)$ \\
Mean (SD) cost of drugs used (HK\$) & $1431(1933)(\mathrm{n}=226)$ \\
\hline${ }^{*}$ Cost per day of hospital stay based on HK hospital authority published figures: \\
HK\$2596 (acute hospital); HK\$1496 (convalescent hospital). Cost per day of oxygen: \\
HK\$20.
\end{tabular}

was non-specific, accompanied by a decrease in general condition, and did not correspond to underlying aetiological agents. However, it led to functional and cognitive impairment, with a mortality of $9.7 \%$. A diagnostic yield of identified organisms of $61.4 \%$ was achieved, facilitated by the use of rapid molecular methods. ${ }^{10}$ Bacterial infection accounted for a higher percentage of ILI than viral infection (53.3\% vs $46.7 \%$ ), and $S$ pneumoniae remained the most common bacterial cause. The incidence of bacterial infections as the cause of ILI was comparable with the weekly incidence of 15-30 cases/1000 residents reported by Harling et al. ${ }^{7}$ The incidence partly reflected the trend of ILI in the community, but there was an underlying background level of ILI episodes that did not fluctuate with the season. From the 1980s onwards, the percentage of subjects with an identified underlying aetiology rose from $21 \%{ }^{13}$ to about $40 \%$ in the 1990 s, ${ }^{14}{ }^{15}$ and up to $64 \%$ in the 2000 s. $^{8}$

In addition to $S$ pneumoniae, $P$ aeruginosa, other Gramnegative bacteria and $S$ aureus (including MRSA) accounted for $19.8 \%$ of all aetiological agents identified. This is not surprising since many subjects have co-morbidities such as chronic airway diseases, whereas some might have aspiration pneumonia where Gram-negative organisms would be more commonly isolated. The data reaffirmed the importance of these multidrug-resistant bacteria in the aetiology of pneumonia in this setting. ${ }^{16}$ Pneumonia in non-ambulatory residents of nursing homes and other long-term care facilities epidemiologically mirrors hospital-acquired pneumonia ${ }^{17}$ and should be treated according to healthcare-associated pneumonia (HCAP) guidelines. ${ }^{18} 19$ Interestingly, atypical organisms including Chlamydophila and Mycoplasma, which are detected only by molecular methods, occurred with comparable frequency to $H$ influenzae. Since 27 ILI episodes (10.4\%) had positive viralbacterial combinations, a proportion of bacterial infection could be superimposed on an initial viral infection with or without aspiration.

Viral aetiology was identified in this study in $46.7 \%$ of cases with positive aetiology, higher than the finding of $43 \%$ in a prospective community cohort surveillance of 533 subjects aged 60-90 years. ${ }^{20}$ The most common viruses in our study were RSV, parainfluenza 1 and 3 and hMPV, with influenza A ranked fifth. The use of the term "ILI" in Hong Kong tends to be equated with influenza A infection, with subsequent infection control implications; perhaps a broader term such as $\mathrm{HCAP}^{18}$ or-more precisely-nursing home acquired pneumonia $(\mathrm{NHAP})^{8}$ would be more appropriate. Previous studies in community dwelling elderly individuals aged $\geqslant 65$ years have 
Table 6 Influenza A antibody titre by vaccination status

\begin{tabular}{clllll}
\hline & $\begin{array}{l}\text { Within } \mathbf{6} \text { months } \\
(\mathbf{n}=\mathbf{1 2 1})\end{array}$ & $\begin{array}{l}\text { Within 12 months } \\
(\mathbf{n}=\mathbf{1 0 8})\end{array}$ & $\begin{array}{l}\text { None } \\
(\mathbf{n}=\mathbf{2 7})\end{array}$ & $\begin{array}{l}\text { Total* } \\
(\mathbf{n}=256)\end{array}$ & p Value \\
\hline Titre interval (2nd serum) & & & & & \\
0 (antibody not detected) & $15(12.4)$ & $10(9.3)$ & $4(14.8)$ & $29(11.3)$ & 0.031 \\
$<10$ & $12(9.9)$ & $25(23.1)$ & $6(22.2)$ & $43(16.8)$ & \\
10 & $22(18.2)$ & $25(23.1)$ & $6(22.2)$ & $53(20.7)$ & \\
20 & $32(26.4)$ & $19(17.6)$ & $7(25.9)$ & $58(22.7)$ \\
40 & $22(18.2)$ & $24(22.2)$ & $2(7.4)$ & $48(18.8)$ \\
80 & $15(12.4)$ & $2(1.9)$ & $2(7.4)$ & $19(7.4)$ & \\
160 & $2(1.7)$ & 0 & 0 & $2(0.8)$ & \\
$\geqslant 320$ & $1(0.8)$ & $3(2.8)$ & 0 & $4(1.6)$ & \\
\hline
\end{tabular}

Data are shown as $\mathrm{n}(\%)$. Three cases were missing.

$p$ value calculated by $\chi^{2}$ test. Ordinal trend measure Somers $D(d=0.155,95 \% \mathrm{Cl} 0.03$ to $0.275, \mathrm{p}=0.013)$.

shown that RSV and hMPV were each responsible for as many hospital admissions for respiratory infection as influenza, ${ }^{21}$ and that $\mathrm{hMPV}$ is a common and ubiquitous human pathogen in children and elderly people, with outbreaks having been described in elderly patients in Japan. ${ }^{22}$

Seasonal variations in ILI episodes were mainly caused by variation in viral infections. Contrary to general assumptions, influenza A infections were more common only in JanuaryMarch, and during this same winter period $S$ pneumoniae caused more ILI episodes while CoV and RSV were equally prevalent. The current policy of influenza A vaccination in late autumn seems appropriate, and the introduction of pneumococcal vaccination in elderly subjects aged $\geqslant 65$ years may reduce further the rates and hospital admissions of these ILI infections. ${ }^{23}$

The clinical features of ILI were non-specific and no particular pattern could be attributed to any particular organism. The presentation is typical of acute illnesses occurring among the frail elderly population, accompanied by functional and cognitive decline and the occurrence of delirium. The impact of infective episodes on function have also been emphasised in other surveys, ${ }^{20} 24$ and reinforces the need to carry out comprehensive multidimensional assessment and rehabilitation in these patients to avoid a rapid spiral towards increasing dependence.

Only MRSA and Klebsiella were significantly associated with mortality; no association was observed for other organisms or the presence of premorbid conditions. The higher frequency of food being withheld among those who died could be a consequence of the severity of illness, or it might represent those who had aspirated as they were at the end of life stage while in the RCHE. The other factors were general features of frailty.

Antibiotics were prescribed in $87 \%$ of cases in this study. The introduction of rapid molecular methods for detecting a wider range of respiratory viruses will enable early de-escalation of antibiotics in patients with clinical improvement and with viral aetiology..$^{10}$ We have recently shown that a combination of two or all three biomarkers (serum procalcitonin, neopterin and Creactive protein) may improve the discriminatory power to delineate bacterial versus viral aetiology in patients presenting with lower respiratory tract infection. ${ }^{26}$ Interestingly, a large proportion of infections caused by $P$ aeruginosa occurred in the summer months of July-September. Further risk factors may be examined to elicit subgroups that may be at risk for such infections. It has been shown that early initiation of appropriate antibiotic therapy improves outcome and reduces mortality. ${ }^{27}$ Thus, adequate cover for these drug-resistant organisms will need to be reviewed for these RCHE cases.

Similar to our observation in patients admitted to hospital for acute exacerbations of chronic obstructive pulmonary disease, ${ }^{28}$ there was no difference in the occurrence of influenza A between those who were vaccinated and those who were unvaccinated in this study, although neither study was designed to address the question of vaccine efficacy. Similarly, there was no marked difference in influenza A antibody titres between those who had received vaccination within 6 months and those who had received vaccination at 6-12 months, perhaps suggesting a longer lasting effect beyond 6 months.

This study was limited by the fact that only four RCHEs near the Prince of Wales Hospital were chosen so that specimens could be sent to the hospital quickly. There might be regional variations in the prevalence of infective organisms, although the pattern in this study is comparable to the CHP sentinel survey of ILI in RCHEs and the general community. Although the sputum pathogens included in the study were those that gave only heavy predominant or pure growth of the organism on cultures, it is recognised that a proportion might still represent colonisation in the background of chronic lung disease or prior use of antibiotics. The analysis of factors associated with mortality was based on univariate analysis and did not take into account the severity of illness and other possible confounding factors since the study was not primarily designed to address this question. In addition, we were not able to carry out direct observation of compliance with infection control policy, relying on reports only. Nevertheless, this represented a detailed clinical and microbiological study of ILI in RCHEs over a 1-year period, where the latest laboratory techniques yielded a very high rate of organism identification and information collected will be useful to guide future clinical management.

In summary, ILI represents a considerable burden of disease in RCHE, with $87 \%$ giving rise to hospital admissions. Clinical presentation of ILI in older people is non-specific and does not correspond to underlying aetiological agents. A large percentage of ILI is due to bacterial and viral infections other than influenza in the RCHE population.

Funding: This project was funded by the Research Fund for the Control of Infectious Diseases (Health and Food Bureau, HKSAR) \#04050452.

Competing interests: None.

Ethics approval: The study was approved by the ethics committee of the Chinese University of Hong Kong and appropriate informed written consent was obtained from the subjects or their immediate relatives.

\section{REFERENCES}

1. Ahmed AH, Nicholson KG, Nguyen-van Tam JS, et al. Effectiveness of influenza vaccine in reducing hospital admissions during the 1989-90 epidemic. Epidemiol Infect 1997:118:27-33.

2. Nichol KL, Nordin JD, Nelson DB, et al. Effectiveness of influenza vaccine in the community-dwelling elderly. N Engl J Med 2007;357:1373-81. 
3. Leung JCK. Effectiveness of influenza vaccination among elderly home residents in Hong Kong: a retrospective cohort study. The HK Practitioner 2007:29:123-33.

4. Nicholson KG, Baker DJ, Farquhar A, et al. Acute upper respiratory tract viral illness and influenza immunization in homes for the elderly. Epidemiol Infect 1990;105:609-18.

5. Falsey AR, Hennessy PA, Formica MA, et al. Respiratory syncytial virus infection in elderly and high risk adults. N Engl J Med 2005;352:1749-59.

6. Nicholson KG, Wood JM, Zambon M. Influenza. Lancet 2003;362:1733-45.

7. Harling R, Hayward A, Watson JM. Implications of the incidence of influenza-like illness in nursing homes for influenza chemoprophylaxis: descriptive study. BMJ 2004:329:663-4.

8. Janssens FP, Krause KH. Pneumonia in the very old. Lancet Infect Dis 2004;4:112-24.

9. Jefferson T. How to deal with influenza? Real time surveillance providing information on circulating agents is the key. BMJ 2004;329:633-4.

10. Lam W, Yeung AC, Tang JW, et al. Rapid multiplex nested-PCR for detection of respiratory viruses. J Clin Microbiol 2007:45:3631-40.

11. Mahoney FI, Barthel DW. Functional evaluation. Barthel index. Maryland State Med J 1965;14:61-5.

12. Woo J, Ho SC, Yu LM, et al. Impact of chronic diseases on functional limitations in elderly Chinese aged 70 years and over: a cross sectional and longitudinal survey. J Gerontol Med Sci 1998;53A:M102-6.

13. Cohen MA, Pang JC. A retrospective study of community-acquired pneumonia in Hong Kong with special reference in the choice of antibiotics. Singapore Med J 1988;30:141-4.

14. Chan HS, Cohen M, Pang J. A prospective study of community-acquired pneumonia in Hong Kong. Chest 1992;101:442-6.

15. Nicholson KG, Kent J, Hammersley V, et al. Acute viral infections of upper respiratory tract in elderly people living in the community: comparative, prospective, population based study of disease burden. BMJ 1997;315:1060-4.

16. El Solh AA, Sikka P, Ramadan F, et al. Etiology of severe pneumonia in the very elderly. Am J Respir Crit Care Med 2001;163:645-51.

17. Mandell LA, Wunderink RG, Anzueto A, et al. Infectious Diseases Society of America/American Thoracic Society consensus guidelines on the management of community-acquired pneumonia in adults. Clin Infect Dis 2007;44(Suppl 2):S27-72.
18. Anon. Guidelines for the management of adults with hospital-acquired, ventilatorassociated, and health-care associated pneumonia. The official statement of the American Thoracic Society and IDSA Guideline Committee, October 2004. Am J Respir Crit Care Med 2005;171:388-416.

19. Anon. Guidelines for preventing health-care associated pneumonia, 2003. Recommendations of $\mathrm{CDC}$ and the Healthcare Infection Control Practices Advisory Committee, CDC. MMWR 2004;53:1-36.

20. Nicholson KG, Kent J, Hammersley V, et al. Risk factors for lower respiratory complications of rhinovirus infections in elderly people living in the community: a prospective cohort study. BMJ 1996;313:1119-23.

21. Kaye M, Skidmore S, Osman H, et al. Surveillance of respiratory virus infections in adult hospital admissions using rapid methods. Epidemiol Infect 2006;134:792-8.

22. Honda H, Iwahashi J, Kashiwagi T, et al. Outbreak of human metapneumovirus infection in elderly inpatients in Japan. J Am Geriatr Soc 2006:54:177-80.

23. Vila-Corcoles A, Ochoa-Gondar 0, Hospital I, et al. Protective effects of the 23valent pneumococcal polysaccharide vaccine in the elderly population: the EVAN-65 study. Clin Infect Dis 2006;43:860-8.

24. High K, Bradley S, Loeb $\mathbf{M}$, et al. A new paradigm for clinical investigation of infectious syndromes in older adults: assessing functional status as a risk factor and outcome measure. J Am Geriatr Soc 2005;53:528-35.

25. Barker W, Falsey A, Walsh E. Functional decline in older patients hospitalized with cardiopulmonary disease associated with influenza A or respiratory syncytial virus. Age Ageing 2004;33(Suppl ii):23.

26. Ip $\mathbf{M}$, Rainer TH, Lee $\mathrm{N}$, et al. Value of serum procalcitonin, neopterin, and $\mathrm{C}$-reactive protein in differentiating bacterial from viral etiologies in patients presenting with lower respiratory tract infections. Diagn Microbiol Infect Dis 2007:59:131-6.

27. Meehan TP, Fine MJ, Krumholz HM, et al. Quality of care, process, and outcomes in elderly patients with pneumonia. JAMA 1997;278:2080-4.

28. Ko FW, Ip M, Chan PK, et al. Viral etiology of acute exacerbations of chronic obstructive pulmonary disease in Hong Kong. Chest 2007;132:900-8. 